\title{
Acute Ischemic Stroke Management: A Clinical Audit in Accordance with the 2019 AHA Guidelines
}

This article was published in the following Dove Press journal:

Clinical Audit

\author{
Muhammad Qasim Bhatti ${ }^{1}$ \\ Richard Mathew Francis' \\ Firas Khaleefah Al Nidawi ${ }^{1,2}$ \\ 'School of Medicine, Royal College of \\ Surgeons in Ireland-Bahrain, Al Sayh, \\ Bahrain; ${ }^{2}$ Department of Neurology, King \\ Hamad University Hospital, Al Sayh, \\ Bahrain
}

\begin{abstract}
Background and Objective: Acute ischemic stroke (AIS) is one of the leading causes for disability worldwide. Incidence has substantially been on the rise globally including Bahrain. The American Heart Association has proposed their latest recommendations with careful integration of the latest literature to optimize stroke care. The aim of this clinical audit is to determine adherence of King Hamad University Hospital to the latest recommendations provided by the AHA/ASA association in order to evaluate the quality of AIS care and highlight improvements to standard practice.
\end{abstract}

Patients and Methods: A retrospective review of patient records was conducted on 75 eligible patients. Patient charts were analyzed for adherence to a select number of parameters pertinent to the AHA/ASA guidelines.

Results: Results indicated high adherence rates ranging from $61.1 \%$ to $100 \%$ for parameters pertaining to stroke scales, imaging, door to needle times, blood glucose, electrocardiograms and anti-platelet agents. Standards were met in the in-patient setting for the relevant guidelines with the exception of dysphagia and dvt prophylaxis.

Conclusion: Dysphagia screening and dvt prophylaxis adherence rates should be improved. Specific recommendations are proposed including checklist implementation, informing patient care staff for prompt dysphagia assessment and integrating latest guidelines into the existing dvt prophylaxis protocol.

Keywords: ischemic, stroke, audit, alteplase, adherence

\section{Introduction}

Acute ischemic stroke (AIS) is one of the most significant causes of morbidity and mortality across the world. Globally, although mortality rates have decreased dramatically over the past two decades, stroke incidence has not which has resulted in a significant burden on health care systems worldwide. ${ }^{1}$ Currently, it is the leading cause of death after cardiovascular disease. According to the World Health Organization, the global burden of stroke continues to rise and approximately 15 million people each year suffer a stroke. ${ }^{2}$ In the United States, approximately 795,000 people per year are affected. ${ }^{3}$ For the Middle East and particularly Bahrain, stroke incidence has been drastically increasing. ${ }^{4}$ Over the past decade, Bahrain has seen an approximate two-fold rise in its incidence. ${ }^{4,5}$ Moreover, presenting patients are of a younger demographic when compared to their Western counterparts suffering from various comorbidities. ${ }^{5}$

In patients that have suffered an acute stroke, the consequences can be severely debilitating resulting in disability and other poor neurologic sequelae. It is therefore imperative that patients undergo adequate management for their symptoms.
Correspondence: Muhammad Qasim Bhatti

Email qb2517@hotmail.com 
Throughout the years, a variety of therapies have revolutionized acute stroke care. These involve the usage of thrombolytic therapy, endovascular thrombectomy, and revascularization methods integrated with efficient neuroimaging, and appropriate clinical evaluation of patients. ${ }^{3,6-9}$ The management of acute ischemic stroke continues to be scrutinized and updated. Currently, evidence-based algorithms and recent findings have been integrated within the AHA/ASA guidelines to further optimize AIS management. For example, findings from Middleton et $\mathrm{al}^{10}$ and Miles et al ${ }^{11}$ assessing dysphagia have been incorporated into these updated guidelines outlining the significance of swallowing dysfunction post stroke and aspiration. The most recent guidelines were published in 2019 which are an update to the 2018 guidelines that provide a thorough comprehensive list of recommendations to clinicians. ${ }^{6-11}$

Due to the aforementioned increase in stroke incidence in Bahrain, optimal early stroke management following a standardized approach is essential in order to lower morbidity and mortality. ${ }^{4,5}$ As King Hamad University hospital is one of the leading stroke centers in Bahrain, the aim of this study is to conduct a clinical audit at the institution in order to review quality of acute ischemic stroke management as per the AHA/ASA guidelines and outline areas of improvement. As the primary stroke center, the emergency and neurologic care services are particularly busy with a high patient intake. This study will allow us to assess the quality of acute ischemic stroke management and will make clinicians aware of any pitfalls in the acute setting. It will help in allowing adequate resource allocation and an optimal standardized approach to stroke management which will eventually reflect on improved patient outcomes and decreased length of stay. Specifically, this study will audit adherence to recommendations in regards to emergency and inpatient care such as diagnostic algorithms for candidate categorization for mechanical thrombectomy or thrombolytic therapy, initial imaging completed, Door to Needle time, blood glucose analysis, baseline ECG, cardiac biomarkers analysis, anti-platelet treatment, stroke units, maintenance of volume/fluid status and blood glucose, screening for dysphagia, nutrition and DVT prophylaxis. ${ }^{6}$ Guidelines in regard to these recommendations have been revised and updated in 2019. ${ }^{6}$ Moreover, we have selected these specific guidelines as they are highly relevant in order to evaluate AIS care from the emergency to the inpatient department.

\section{Patients and Methods}

The setting of the clinical audit was the emergency and inpatient neurologic care at the King Hamad University Hospital located in Al Sayh, Bahrain. Our inclusion criteria involved all patients that were managed and admitted for acute ischemic stroke from January 1st, 2019 to January 1st, 2020. These patients were identified through a retrospective chart review by accessing the hospital's electronic medical health records. Through reviewing patient records, 283 patients had presented with symptoms similar to stroke in the emergency department, out of which 75 were included in the study. The remainder were excluded as they did not match the inclusion criteria; these patients were either diagnosed with other neurologic pathologies or were admitted and managed elsewhere. Figure 1 describes the inclusion criteria.

Electronic medical health records were analyzed for adherence to a select number of parameters from the recommendations as adapted from the AHA guidelines. ${ }^{6}$ The rationale and relevance of these specific parameters has been well elucidated in Table 1.

We assessed the adherence of each variable for each patient. If a specific parameter was not mentioned in the patient's chart, we assumed that it was not completed. Data were collected nominally on a Microsoft Excel 2020 Spreadsheet and analyzed for percentage adherence to each data point. The project was ethically approved from the Institutional Review Board at King Hamad University Hospital, Bahrain. Since this is a retrospective chart review, patient consent was not required and was waived by the Institutional Review Board. All patient data accessed complied with the relevant data protection and privacy regulations. All data were analyzed in anonymized form. Only pooled anonymized data is displayed.

\section{Results}

Total number of patients diagnosed with AIS was 75. The hospital implements using stroke scales primarily NIHSS. NIHSS was completed for $70.7 \%$ of patients. For initial imaging, $100 \%$ of patients underwent either a non-contrast CT or MRI before the initiation of any therapy. Certain patients had additional use of various imaging modalities including CT angiography or a CT perfusion scan to assess eligibility for mechanical thrombectomy; however, this was on case-specific basis and was not focused upon in this study. 18 out of the total 75 patients received 

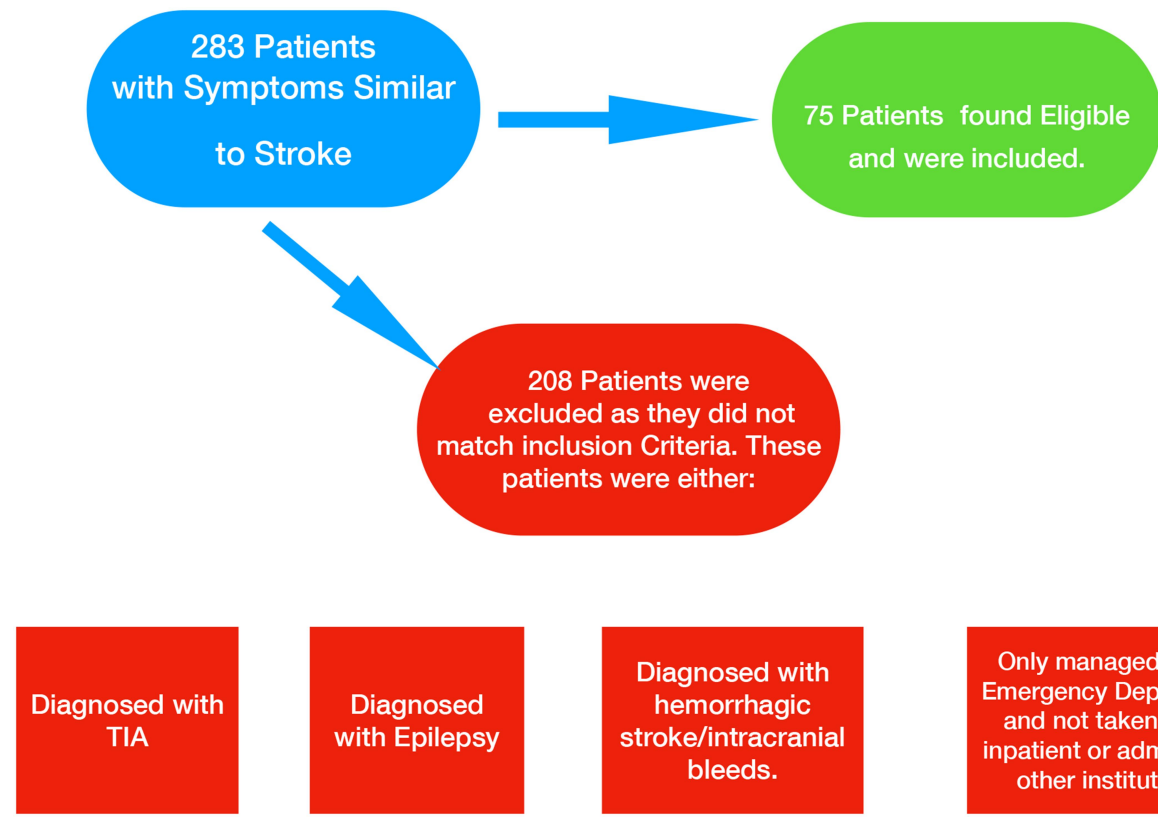

Diagnosed with

hemorrhagic

stroke/intracranial bleeds.
Only managed at the Emergency Department and not taken as an inpatient or admitted at other institutions.

Figure I Algorithm outlining patient selection.

thrombolytic therapy. From the patients that received IV alteplase/tPA, door-to-needle time was less than $60 \mathrm{~min}$ utes for $61.1 \%$ of patients (Table 2).

Our results indicated that blood glucose was tested in $100 \%$ of patients along with other hematological tests namely a complete coagulation profile (PT, aPTT, INR, etc.) and other lab tests including a lipid profile. Baseline ECG was completed in $100 \%$ of patients. Cardiac markers including baseline troponin levels were evaluated in 13.3\% of patients (Table 3).

Administration of anti-platelet agents was also assessed. Anti-platelet treatment was delayed for the first 24 hours for those patients that had received IV alteplase therapy. Moreover, $69.3 \%$ of patients only received dual anti-platelet therapy for their acute stroke presentation (Table 3).

Table I Audited Variables

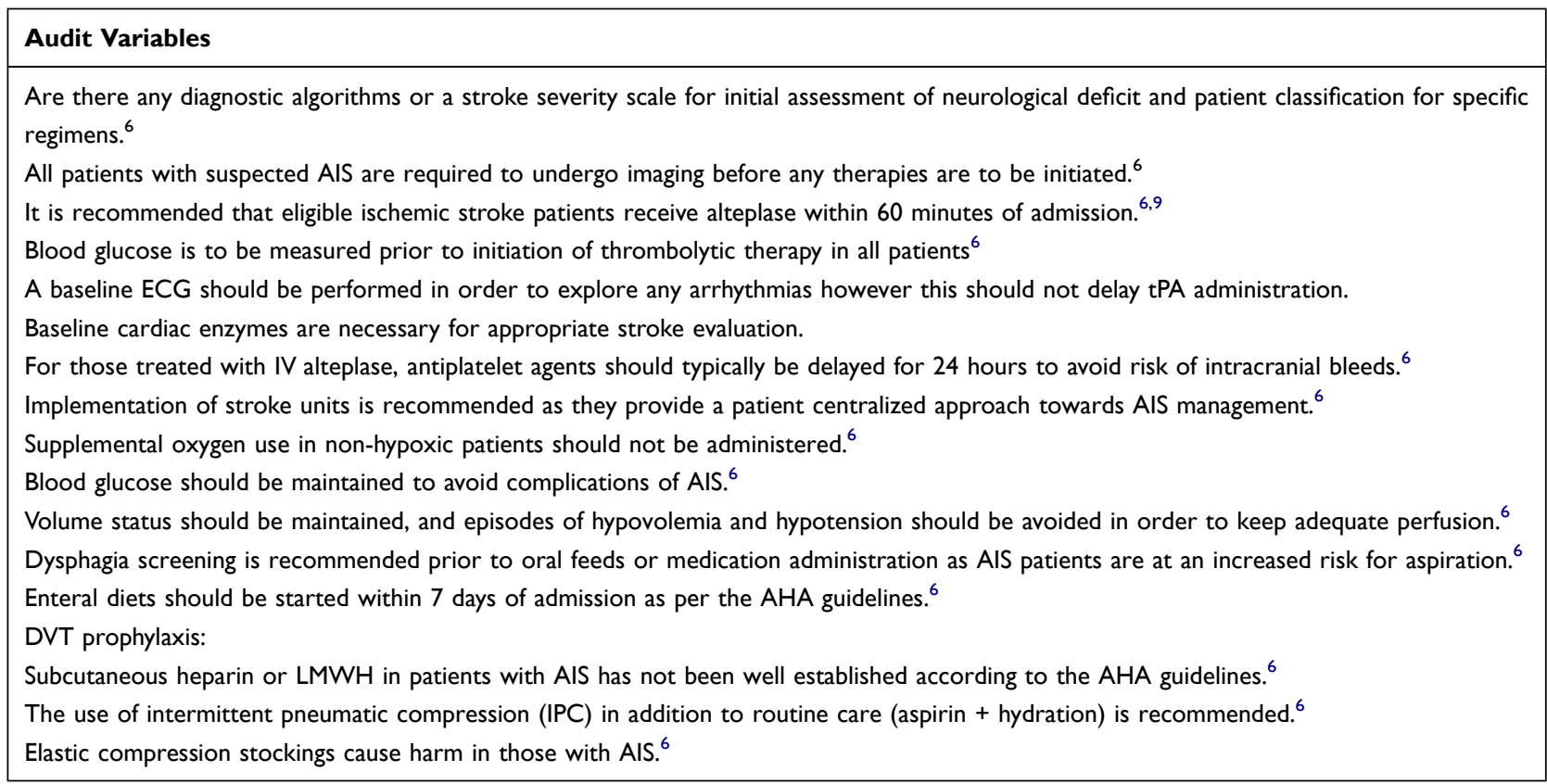


Table 2 Results for Parameters Assessed in Emergency Care

\begin{tabular}{|l|l|l|}
\hline Audit Parameter & $\begin{array}{l}\text { Proportion of } \\
\text { Patients in } \\
\text { Which } \\
\text { Completed (P) } \\
\text { and Percentage }\end{array}$ & $\begin{array}{l}\text { Number of Health } \\
\text { Records Assessed } \\
\text { for Relevant } \\
\text { Parameter (N) }\end{array}$ \\
\hline $\begin{array}{l}\text { Diagnostic Algorithm/ } \\
\text { Stroke Scale (NIHSS) }\end{array}$ & $\begin{array}{l}\mathrm{P}=53 / 75 \\
70.7 \%\end{array}$ & $\begin{array}{l}\mathrm{N}=75 \text { (All patients } \\
\text { with AIS) }\end{array}$ \\
\hline $\begin{array}{l}\text { CT/MRI prior } \\
\text { treatment }\end{array}$ & $\begin{array}{l}\mathrm{P}=75 / 75 \\
100 \%\end{array}$ & $\begin{array}{l}\mathrm{N}=75 \text { (All patients } \\
\text { with AIS) }\end{array}$ \\
\hline $\begin{array}{l}\text { Door-to-Needle }<60 \\
\text { mins }\end{array}$ & $\begin{array}{l}\mathrm{P}=11 / 18 \\
61.1 \%\end{array}$ & $\begin{array}{l}\mathrm{N}=18 \text { (All patients } \\
\text { eligible for tPA) }\end{array}$ \\
\hline
\end{tabular}

Table 3 Results for Parameters Pertaining to Other Diagnostic Tests and Anti-Platelet Therapy

\begin{tabular}{|l|l|l|}
\hline Audit Parameter & $\begin{array}{l}\text { Proportion of } \\
\text { Patients in } \\
\text { Which } \\
\text { Completed } \\
\text { (P) and } \\
\text { Percentage }\end{array}$ & $\begin{array}{l}\text { Number of } \\
\text { Health Records } \\
\text { Assessed for } \\
\text { Relevant } \\
\text { Parameter (N) }\end{array}$ \\
\hline $\begin{array}{l}\text { Blood Glucose } \\
\text { Measurement }\end{array}$ & $\begin{array}{l}\mathrm{P}=75 / 75 \\
100 \%\end{array}$ & $\begin{array}{l}\mathrm{N}=75 \text { (All patients } \\
\text { with AIS) }\end{array}$ \\
\hline Baseline Electrocardiogram & $\mathrm{P}=75 / 75$ & $\begin{array}{l}\mathrm{N}=75 \text { (All patients } \\
\text { with AIS) }\end{array}$ \\
\hline $\begin{array}{l}\text { Cardiac Enzymes (Baseline } \\
\text { Troponins) }\end{array}$ & $\begin{array}{l}\mathrm{P}=10 / 75 \\
13.3 \%\end{array}$ & $\begin{array}{l}\mathrm{N}=75 \text { (All patients } \\
\text { with AIS) }\end{array}$ \\
\hline $\begin{array}{l}\text { Anti-platelets delayed for } \\
24 \text { hours in patients } \\
\text { treated with alteplase }\end{array}$ & $\begin{array}{l}\mathrm{P}=18 / 18 \\
100 \%\end{array}$ & $\begin{array}{l}\mathrm{N}=18 \text { (All patients } \\
\text { who received } \\
\text { alteplase therapy) }\end{array}$ \\
\hline
\end{tabular}

Our results indicated that the hospital implemented stroke units in $100 \%$ of patients. Supplement oxygen was only used in hypoxic patients which accounted for approximately $13.5 \%$ of patients from our data set. Blood glucose levels were adequately maintained for all patients. $45.3 \%$ of patients approximately were kept on an insulin sliding scale regimen as required. Blood pressure levels were appropriately maintained in all patients with no episodes of hypovolemia or hypotension. Dysphagia screening was conducted in $1.3 \%$ of patients. $96 \%$ of patients were started on enteral feeds within one week. In terms of DVT prophylaxis, $20 \%$ of patients received intermittent pneumatic compression stockings in addition to routine care, $9.3 \%$ patients received subcutaneous clexane, $9.3 \%$ subcutaneous heparin and $22.6 \%$ patients were on compression/TED stockings (Table 4).

\section{Discussion}

The primary objective of this clinical audit was to investigate acute ischemic stroke (AIS) care and outline areas where patient care can be improved. Utilization of stroke severity scales is in compliance with the AHA guidelines; however, the adherence rate for the implementation of NIHSS scale is $70.7 \%$. This discrepancy may be attributed to a lack of reporting on patient charts. As King Hamad University Hospital is a dedicated stroke center, their code stroke protocol includes an initial NIHSS evaluation. However, this needs to be manually recorded on the electronic medical chart by the relevant resident which opens doors for reporting bias. As this is a retrospective chart review, reporting bias is always a possibility. Imaging before any treatment initiation is necessary to exclude intracranial hemorrhages and to determine eligibility for thrombolysis or mechanical thrombectomy. ${ }^{6} \mathrm{CT}$ or MRI scans were performed prior to therapy in all patients with AIS. 18 out of 75 patients received alteplase therapy. The rest of the patients were found either to be out of the window period for thrombolysis or had a contraindication to thrombolysis. From the patients that received alteplase, door-to-needle time was $<60$ minutes for $61.1 \%$ of patients which suggests a good adherence to the AHA recommendation that door-to-needle times be kept less than 60 minutes. In 2010, the AHA/ASA outlined the Target: Stroke initiative whose initial goal recommended to keep the door-to-needle time within 60 minutes in at least $50 \%$ of eligible patients presenting with AIS. ${ }^{12}$ Although the stroke program at the hospital meets the initial goal, there is always room for improvement. Various centers all over the US and Europe have drastically reduced their door-toneedle times and have surpassed the initial goal through efficient implementation of effective strategies. ${ }^{13}$ It should be reiterated that alteplase therapy is time-dependent and prompt initiation leads to decreased morbidity and mortality. ${ }^{6}$ The hospital already employs a robust code stroke protocol; however, strategies that proved beneficial in its western counterparts should be identified and implemented to further attempt to improve their code stroke program.

Blood glucose measurement should be completed prior to tPA therapy. ${ }^{6}$ Results showed complete compliance to this recommendation. Blood glucose is vital to assess as severe hypoglycemia can mimic stroke symptoms. Baseline electrocardiograms standards were met in all patients. Moreover, as reflected by the aforementioned 
Table 4 Results for Other Parameters Assessed for in-Patient Care

\begin{tabular}{|c|c|c|}
\hline Audit Parameter & $\begin{array}{l}\text { Proportion of Patients in Which } \\
\text { Completed (P) and Percentage }\end{array}$ & $\begin{array}{l}\text { Number of Health Records Assessed for } \\
\text { Relevant Parameter (N) }\end{array}$ \\
\hline Stroke Units/Code stroke protocol & $\begin{array}{l}P=75 / 75 \\
100 \%\end{array}$ & $\mathrm{~N}=75$ (All patients with AIS) \\
\hline $\begin{array}{l}\text { Supplemental Oxygen not recommended } \\
\text { in non-hypoxic patients }\end{array}$ & $\begin{array}{l}P=75 / 75 \\
100 \% \\
\text { Patients only given oxygen when hypoxic }\end{array}$ & N=75 (All patients with AIS) \\
\hline $\begin{array}{l}\text { Correction and maintenance of blood } \\
\text { glucose }\end{array}$ & $\begin{array}{l}P=75 / 75 \\
100 \%\end{array}$ & $\mathrm{~N}=75$ (All patients with AIS) \\
\hline $\begin{array}{l}\text { Maintenance of blood pressure and } \\
\text { volume status }\end{array}$ & $\begin{array}{l}P=75 / 75 \\
100 \%\end{array}$ & $\mathrm{~N}=75$ (All patients with AIS) \\
\hline Dysphagia Screening & $\begin{array}{l}\mathrm{P}=1 / 75 \\
\mathrm{I} .3 \%\end{array}$ & $\mathrm{~N}=75$ (All patients with AIS) \\
\hline Enteral Feeds & $\begin{array}{l}P=72 / 75 \\
96 \%\end{array}$ & $\mathrm{~N}=75$ (All patients with AIS) \\
\hline DVT Prophylaxis & $\begin{array}{l}\text { IPC+ routine care } P=15 / 75 \\
20 \% \\
\text { Subcutaneous Heparin } P=7 / 75 \\
9.3 \% \\
\text { Subcutaneous Clexane } P=7 / 75 \\
9.3 \% \\
\text { TED/Elastic Compression Stockings } P=17 / 75 \\
22.6 \%\end{array}$ & $\mathrm{~N}=75$ (All patients with AIS) \\
\hline
\end{tabular}

door-to-needle times, it is unlikely that this delayed tPA administration. For a comprehensive work-up of AIS, limited data also suggest baseline troponin evaluation in inpatient care. ${ }^{6}$ However, our results indicated that this was based on a clinician's judgment wherever a myocardial infarction was suspected.

For patients that received alteplase therapy, aspirin was not administered for the first 24 hours meeting the criteria set for anti-platelet agents. Findings of the ARTIS trial demonstrated an increased risk of intracranial hemorrhage following early IV aspirin administration in tPA patients. $^{6,14}$

Implementation of stroke units, oxygen use only in hypoxia, maintenance of blood pressure and volume status were all adequately achieved. In order to maintain appropriate perfusion, episodes of hypovolemia and hypotension need to be prevented. ${ }^{6}$ A study by Wohlfahrt et $\mathrm{al}^{15}$ demonstrated an association of significantly elevated mortality rates in patients with low blood pressures. Enteral diet initiation within 7 days of admission was achieved for the majority of patients exhibiting strong adherence to recommendations.
Various studies have exhibited better patient outcomes in patients where enteral diets were started within the first week. ${ }^{6}$ A particular parameter where adherence was extremely low was dysphagia screening. Even though aspiration precautions were mentioned, there was no screening tool implemented. Dysphagia is a common complication post-AIS and may lead to aspiration pneumonia. ${ }^{16}$ A study by Joundi et $\mathrm{al}^{17}$ demonstrated that patients that failed dysphagia assessment were at greater risk for developing pneumonia, ending up in a long-term care facility, or have severe disability. Screening therefore may prove beneficial to identify these high-risk patients and improve patient outcomes. DVT prophylaxis also exhibited relatively low adherence. Intermittent Pneumatic Compression devices in addition to routine care should take precedent over all other methods of prophylaxis in immobilized AIS patients without any contraindications. ${ }^{6}$ Certain contraindications include but are not limited to suspected DVT, severe congestive heart failure, peripheral vascular disease and inflammatory skin disorders. ${ }^{6}$ Moreover, medical DVT prophylaxis such as the use of 
subcutaneous unfractionated heparin is not well established in AIS patients. ${ }^{6}$ It may have a benefit in certain patients that have a history of thromboembolic disease but there is no method to appropriately stratify the benefit of thromboembolism prevention versus the risk of intracranial hemorrhage. ${ }^{6}$ As this was used in a minority of patients, a physician's clinical judgment may also be a contributing factor. Finally, elastic compression stockings should not be used in ischemic stroke patients however this was not followed. Evidence suggests elastic compression stockings in ischemic stroke patients can actually be harmful. ${ }^{6}$ Clinicians also prescribed clexane (enoxaparin sodium) which is considered a low molecular weight heparin as part of their DVT prophylaxis regimen. This can be attributed to the fact that the hospital has an internal DVT prophylaxis policy.

Certain limitations that should be reiterated are reporting bias. As this is a retrospective study reporting bias is always a possible caveat. This is particularly evident in terms of data abstraction when certain items or data points may not have been recorded in the patient charts. In terms of selection bias, we established distinct inclusion criteria to aim to gather an appropriate representative sample.

\section{Recommendations}

Based on the above findings, certain recommendations can be proposed to current practice in order to improve patient care. Appropriate and active documentation of stroke severity scores should be updated in all patient charts. Specific strategies aimed at further improving door to needle times should be identified and their impact can be assessed. A checklist can be implemented pertaining to parameters related to blood tests, blood glucose, coagulation profile and baseline troponin evaluation along with other routine hematological tests.

To address dysphagia screening, patient care staff should be promptly informed to utilize a screening tool in their assessment. Various bedside screening approaches are available and should be applied. Of note, the Gugging Swallowing Screen is a reliable clinical tool that grades swallowing dysfunction and classifies patients at risk of aspiration. ${ }^{18}$

Similarly, patient care staff should be notified of promoting the use of intermittent pneumatic compression stockings in addition to routine care in immobile patients after assessment of any contraindications. Elastic compression stockings should not be used. DVT prophylaxis protocol policy should be updated to reflect these recommendations in AIS patients.

A re-audit should be conducted to assess if proposed changes to current standards were implemented and their impact on quality of patient care.

\section{Conclusion}

AIS care services pertaining to efficient imaging, door-toneedle times, anti-platelet therapy indications, utilization of stroke units, blood glucose, blood pressure and volume status, supplemental oxygen and enteral feeding all were highly adherent to the recommended parameters. Physicians should be encouraged to perform prompt dysphagia screening into their clinical evaluations post AIS. Moreover, DVT prophylaxis protocols need to be refined to incorporate adherence to latest recommendations in regard to intermittent pneumatic compression stockings when appropriate and to not use elastic compression stockings in AIS patients.

\section{Abbreviations}

AIS, Acute Ischemic Stroke; tPA, tissue plasminogen activator; TIA, transient ischemic attack; DVT, deep vein thrombosis; IPC, intermittent pneumatic compression.

\section{Acknowledgments}

Ethics approval for the study was obtained from the Institutional Review Board at King Hamad University Hospital, Bahrain. The approval reference number for the study is $20-312$.

\section{Disclosure}

The authors have no conflicts of interest to report for this work.

\section{References}

1. Johnson CO, Nguyen M, Roth GA, GBD 2016 Stroke Collaborators. Global, regional, and national burden of stroke, 1990-2016: a systematic analysis for the global burden of disease study 2016. Lancet Neurol. 2019;18(5):439-458. doi:10.1016/S1474-4422(19)30034-1

2. Douglas DR, Luoma V, Reddy U. Acute management of ischaemic stroke. Anaesth Intensive Care Med. 2020;21(1):1-7. doi:10.1016/j. mpaic.2019.10.013

3. Hasan TF, Rabinstein AA, Middlebrooks EH, et al. Diagnosis and management of acute ischemic stroke. Mayo Clinic Proc. 2018;93 (4):523-538. doi:10.1016/j.mayocp.2018.02.013

4. El-Hajj M, Salameh P, Rachidi S, Hosseini H. The epidemiology of stroke in the Middle East. Eur Stroke J. 2016;1(3):180-198. doi:10.1177/2396987316654338

5. Al Banna M, Baldawi H, Khadim A, et al. Stroke in Bahrain: rising incidence, multiple risk factors, and suboptimal care. Int J Stroke. 2015;10(4):615-618. doi:10.1111/ijs.12513 
6. Powers WJ, Rabinstein AA, Ackerson T, et al. Guideline for the early management of patients with acute ischemic stroke: 2019 update to the 2018 guidelines for the early management of acute ischemic stroke: a guideline for healthcare professionals from the American Heart Association/American Stroke Association. Stroke. 2019;50(12):e344 e418.

7. The National Institute of Neurological Disorders and Stroke r-tPA Stroke Study Group. Tissue plasminogen activator for acute ischemic stroke. $N$ Engl J Med. 1995;333(24):1581-1587. doi:10.1056/ NEJM199512143332401

8. Hacke W, Kaste M, Bluhmki E, et al. Thrombolysis with alteplase 3 to 4.5 hours after acute ischemic stroke. $N$ Engl J Med. 2008;359 (13):1317-1329. doi:10.1056/NEJMoa0804656

9. Wardlaw JM, Murray V, Berge E, Del Zoppo GJ. Thrombolysis for acute ischemic stroke. Cochrane Database Syst Rev. 2014;7: CD000213.

10. Middleton S, McElduff P, Ward J, et al. Implementation of evidencebased treatment protocols to manage fever, hyperglycaemia, and swallowing dysfunction in acute stroke (QASC): a cluster randomized controlled trial. Lancet. 2011;378(9804):1699-1706. doi:10.1016/S0140-6736(11)61485-2

11. Miles A, Zeng IS, McLauchlan H, Huckabee ML. Cough reflex testing in dysphagia following stroke: a randomized controlled trial. $J$ Clin Med Res. 2013;5(3):222-233.

12. Kruyt N, Nederkoorn P, Dennis M, et al. Door-to-needle time and the proportion of patients receiving intravenous thrombolysis in acute ischemic stroke. Stroke. 2013;44(11):3249-3253. doi:10.1161/ STROKEAHA.113.001885
13. Kamal N, Holodinsky J, Stephenson C, et al. Improving door-toneedle times for acute ischemic stroke: effect of rapid patient registration, moving directly to computed tomography, and giving alteplase at the computed tomography scanner. Circ Cardiovasc Qual Outcomes. 2017;10(1):e003242. doi:10.1161/CIRCOUTCOMES. 116.003242

14. Zinkstok SM, Roos YB. Early administration of aspirin in patients treated with alteplase for acute ischemic stroke: a randomized controlled trial. Lancet. 2012;380(9843):731-737. doi:10.1016/S01406736(12)60949-0

15. Wohlfahrt P, Krajcoveichova A, Jozifova M, et al. Low blood pressure during the acute period of ischemic stroke is associated with decreased survival. J Hypertens. 2015;33(2):339-345. doi:10.1097/ НJH.0000000000000414

16. Cohen DL, Roffe C, Beavan J, et al. Post-stroke dysphagia: a review and design consideration for future trials. Int J Stroke. 2016;11 (4):399-411. doi:10.1177/1747493016639057

17. Joundi RA, Martino R, Saposnik G, et al. Predictors and outcomes of dysphagia screening after acute ischemic stroke. Stroke. 2017;48 (4):900-906. doi:10.1161/STROKEAHA.116.015332

18. Trapl M, Enderle P, Nowotny M, et al. Dysphagia bedside screening for acute-stroke patients: the gugging swallowing screen. Stroke. 2007;38(11):2948-2952. doi:10.1161/STROKEAHA.107.483933
Clinical Audit

\section{Publish your work in this journal}

Clinical Audit is an international, peer-reviewed, open access journal focusing on the processes and outcomes of clinical audit in any area of healthcare. All aspects of patient care are addressed within the journal and practitioners from all disciplines are invited to submit their work. Areas covered include: Publication of audits; How an audit has changed practice; Practical tips on how to do audits and to

Submit your manuscript here: https://www.dovepress.com/clinical-audit-journal

\section{Dovepress}

avoid pitfalls; How audits have changed patient care; Calls and justifications for new audits. The manuscript management system is completely online and includes a very quick and fair peer-review system, which is all easy to use. Visit http://www.dovepress.com/ testimonials.php to read real quotes from published authors. 